\title{
What are Urban Planning Academics Talking About on Twitter?
}

\author{
Thomas W. Sanchez ${ }^{1}$ (1) \\ ${ }^{1}$ Urban Affairs and Planning, Virginia Tech \\ Keywords: urban planning, twitter, social media
}

https://doi.org/10.32866/001c.23716

Findings

The purpose of this analysis is to report on the topics being discussed by urban planning academics on Twitter. Analyzing the content of the tweets helps to understand the topics of conversation and in some sense, the reasons why planning academics use the platform professionally. In many cases the topics identified reflect the research interests of planning academics, while others extend beyond scholarly activities.

\section{Questions}

What are urban planning academics talking about on Twitter? The following analysis is the first to answer this question by examining the content of tweets for the 12-years period from March 2007 to April 2019. This helps to understand the general topics of conversation and in some ways, the reasons why planning academics use the platform. Previous analyses have focused on how Twitter can be used by urban planners, such as for public participation (see Evans-Cowley and Griffin 2012) and workshops or conferences (see Williamson and Ruming 2018) but did not include planning academics. The following briefly describes the methodology, followed by the results, and concludes with some brief comments.

\section{Methods}

To examine Twitter content exchanged by planning academics, data for this analysis were obtained from two primary sources. A list of 1,104 urban planning faculty from 106 universities across the U.S. and Canada maintained by Sanchez (see Sanchez 2017), were searched on Twitter to find their accounts. Only accounts being used for professional purposes were of interest, so account profiles needed to: a) contain a professional title (e.g., professor), b) mention urban planning or related area of expertise (e.g., transportation, housing, environment), or c) mention an employer's name (i.e., university name or department). In a small number of cases, accounts were included when these criteria were not met but the content of tweets was primarily related to urban planning topics.

A total of 322 Twitter accounts were identified from the list of 1,104 urban planning faculty. For each account, the profiles, tweets, followers, and friends were obtained through the Global Event and Trend Archive Research Project (GETAR) at Virginia Tech ${ }^{1}$. This included all account activity from March 


\begin{tabular}{|c|c|c|c|c|}
\hline Hashtag & $\begin{array}{r}\text { Has } \\
\text { Frequ }\end{array}$ & & $\begin{array}{c}\text { Topic } \\
\text { Frequency }\end{array}$ & \multirow{5}{*}{$\begin{array}{l}\text { Topic } \\
\begin{array}{|l}\text { Analysis } \\
\text { Class } \\
\text { Conference }\end{array}\end{array}$} \\
\hline data & 410 & 410 & & \\
\hline technicity & 526 & 526 & & \\
\hline TRBam & 374 & \multirow{2}{*}{684} & & \\
\hline ASPA2019 & 310 & & & \\
\hline climatechange & 727 & \multirow{2}{*}{1140} & & \multirow{2}{*}{ Climate } \\
\hline climate & 413 & & & \\
\hline NYC & 323 & \multirow{2}{*}{612} & & \multirow{2}{*}{ Place } \\
\hline Toronto & 289 & & & \\
\hline planning & 724 & \multirow{9}{*}{4238} & & \multirow{9}{*}{ Planning } \\
\hline cities & 707 & & & \\
\hline urbanplanning & 661 & & & \\
\hline urban & 442 & & & \\
\hline resilience & 396 & & & \\
\hline sustainability & 347 & & & \\
\hline justsustainabilitie & 342 & & & \\
\hline housing & 335 & & & \\
\hline gentrification & 284 & & & \\
\hline smartcities & 616 & \multirow{3}{*}{1515} & & \multirow{3}{*}{ Technology } \\
\hline bigdata & 482 & & & \\
\hline opendata & 417 & & & \\
\hline
\end{tabular}

Figure 1. Twenty most popular hashtags

2007 to April 2019. Tweet topics were identified by analyzing hashtags and tweet text, but not images, weblinks, or mentions. Tweets often include abbreviations, slang, and jargon, with abbreviations being commonly used due to the 140-character limit on tweets which represents one challenge to detecting tweet topics. Tweets can be original content and recirculated information and of the 258,872 tweets from urban planning faculty analyzed here, nearly half were original content and the other half being retweets. Regardless of type, the content was analyzed to identify tweet topics using hashtag frequency and a topic modeling process for text content. ${ }^{2}$

\section{Findings}

Of the 258,872 total tweets, only about $25 \%(66,179)$ used hashtags, resulting in a total of 100,569 hashtags. There were 27,354 unique hashtags with 9,330 of those being used more than once. The most frequently used hashtags were \#climatechange, \#planning, \#cities, \#urbanplanning, and \#smartcities (see Figure 1). The hashtag frequencies are only for individual appearances and do not account for hashtags used in combinations, so while \#urbanplanning was the fourth most frequently used hashtag, \#planning (724 appearances) and \#urban (442 appearances) may have been used in combination and intended to have the same meaning. 


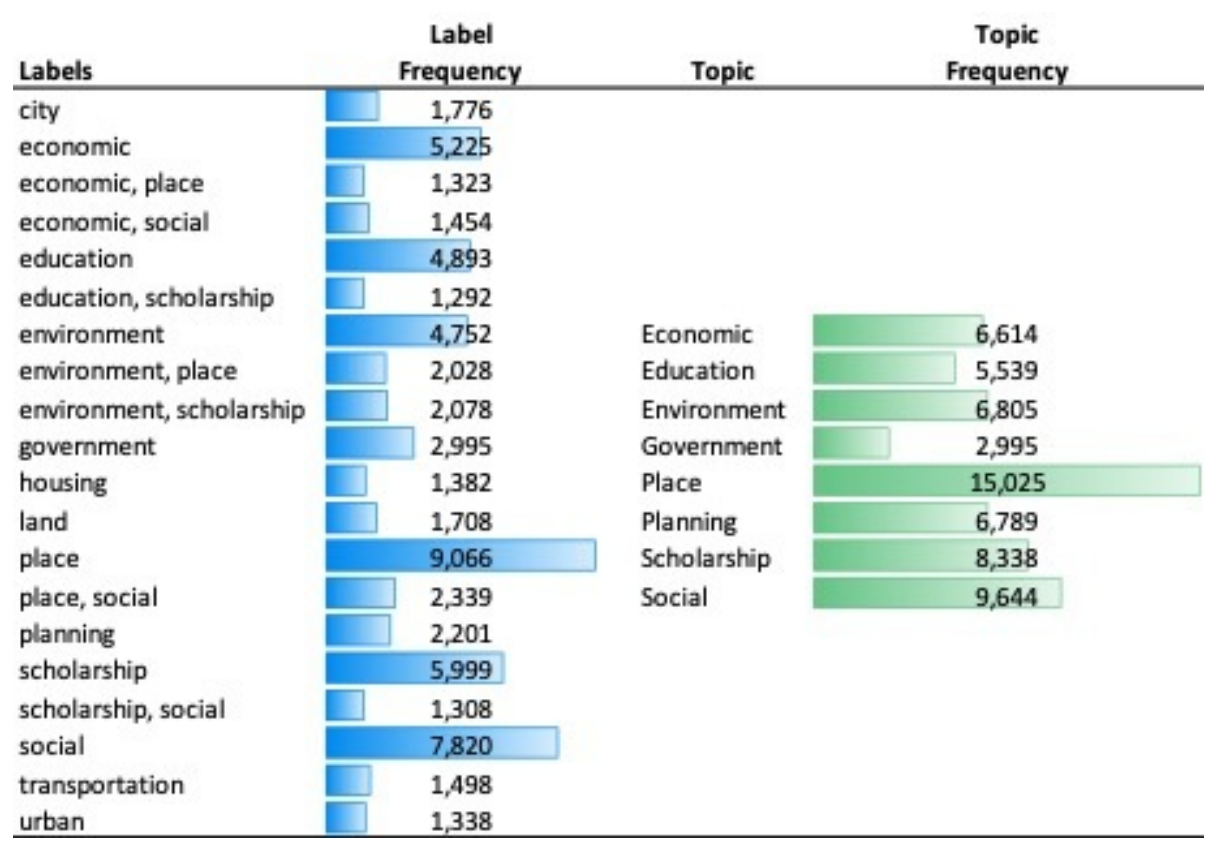

Figure 2. Twenty frequent tweet topics (multi-label)

The hashtags in Figure 1 appeared across a relatively small proportion of all tweets analyzed, with none exceeding $1 \%$ and the top 20 representing less than $9 \%$ of all tweets. Of these 20, 9 were traditional planning-related topics, with the rest being related to technology (3); conferences, climate, and places ( 2 each); and analytics and an online course (1 each). As mentioned earlier, hashtags were not used in a majority of tweets, so it may be a less than ideal way to characterize overall tweet themes. Nonetheless, these hashtags (and topics) provide a general indicator of popular topics.

The second way to examine topics is using tweet text and the frequency of terms. Nearly all of the tweets contained text and were mined to extract frequently occurring terms. The most frequent planning related labels were "place" (3.5\%), "social” (3.0\%), "scholarship" (2.3\%), and "economic" (2.0\%), for a total of 28,110 tweets or about $11 \%$ (see Figure 2).

In terms of the overall frequency of individual labels, the top 20 represented most of those appearing in the multiple label categories listed in Figure 2. The most frequent single term was "place", followed by "social", "scholarship", "economic", "education", and "environment". These terms represent clusters of related unigrams and bigrams analyzed during the text mining process. For instance, "place" includes terms like United States, Canada, Toronto, California, and New York City. The label "social” includes terms like people, women, kids, children, and family. Unlike hashtags, these labels represent groups of related terms. Grouping the labels (e.g., housing + land + planning + transportation $=$ planning) produces a very similar ranking to the ungrouped single labels in Figure 2. Because two labels could be assigned to a topic, the 
frequency was split between terms for the topic level summary. For example, Economic $(6,614=5,225+1,323 / 2+1,454 / 2)$. Except for "scholarship," the eight topics listed are typical urban planning teaching and research topics.

\section{Conclusion}

The list of topics generated by hashtags and tweet text gives a general sense of what urban planning academics are communicating about on Twitter. There are some noticeable differences in topics identified by hashtags and those from the text content of tweets - partly having to do with how hashtags and text are used within tweets. The resulting lists of topics highlight familiar urban planning topics in addition to mentions of professional activities such as academic conferences, scholarly publications, and other research activities. As might be expected, planning academic's Twitter conversations reflect their primary interests in 'places', such as urban areas, communities, neighborhoods, or specific cities. Their tweets also reflect their interests in particular aspects of places, such as the social, economic, and environmental dimensions of those places. And like other academics on Twitter, planning academics talk about scholarship, both their own (aka self-promotion) and that of other academics with common interests.

One question that was of interest that could not be answered was whether planning academics and planning practitioners were communicating with each other on Twitter. Unfortunately, the level of Twitter participation by professional planners is unknown and it is likely they do not tweet in their official capacity with public and private planning organizations. Therefore it is difficult to identify them. This is a topic for future research. Finally, it should be noted that the tweets analyzed here were from before the COVID-19 pandemic, which likely became a very important (if not the most important) Twitter topic among planning academics. This too will be the topic of future analysis.

\section{Acknowledgement}

The author would like to thank Ziqian (Alice) Song for her assistance with Twitter data acquisition. 


\section{REFERENCES}

Evans-Cowley, J. S., and G. Griffin. 2012. "Microparticipation with Social Media for Community Engagement in Transportation Planning." Transportation Research Record 2307 (1): 90-98.

Sanchez, Thomas W. 2017. "Faculty Performance Evaluation Using Citation Analysis: An Update." Journal of Planning Education and Research 37 (1): 83-94. https://doi.org/10.1177/ $\underline{0739456 \times 16633500 .}$.

Sanchez, Thomas W., and Nader Afzalan. 2017. "Mapping the Knowledge Domain of Urban Planning." In Planning Knowledge and Research, edited by Thomas W. Sanchez, 69-84.

Routledge. https://doi.org/10.4324/9781315308715-6.

Williamson, W., and K. J. Ruming. 2018. "Live Tweeting the Planning Reform Workshop." Australian Planner 55 (1): 1-11. 